\title{
Higher follicular fluid glycodelin levels are negatively correlated with embryonic development in assisted reproduction
}

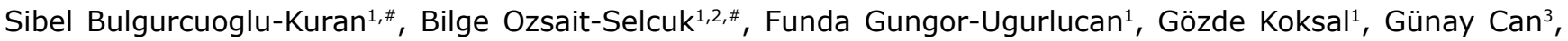 \\ Faruk Buyru ${ }^{1}$
}

${ }^{1}$ Division of Reproductive Endocrinology and Infertility, Department of Obstetrics and Gynecology, Istanbul Medical Faculty, Istanbul University, Istanbul, Turkey

2 Department of Genetics, Aziz Sancar Institute for Experimental Medicine, Istanbul University, Istanbul, Turkey

${ }^{3}$ Department of Public Health, Cerrahpasa Medical Faculty, Istanbul University (Cerrahpasa), Istanbul, Turkey

\#These authors contributed equally to this work.

\begin{abstract}
Objective: To investigate the possible effect of follicular fluid glycodelin levels on the quality of developing oocytes and subsequent in vitro embryo development.

Methods: Follicular fluid glycodelin levels of 145 patients undergoing assisted reproductive treatment were analyzed and the correlation between glycodelin levels and ART outcomes were evaluated.

Results: We found that glycodelin levels were negatively correlated with the number of high quality embryos on day $3(r=-0.20, p=0.05)$. Additionally, higher glycodelin levels were correlated with higher FSH levels $(r=0.18$, $p=0.04)$. However, glycodelin levels were not predictive for implantation $(p=0.67)$ or ongoing pregnancy rates $(p=0.99)$.

Conclusion: Glycodelin in the follicular environment might be one of the factors that influence the competence of growing oocytes and affect the quality of subsequent in vitro embryo development.
\end{abstract}

Keywords: glycodelin, follicular fluid, embryonic development, FSH, assisted reproduction

\section{INTRODUCTION}

The follicular microenvironment of the oocyte is composed of theca and granulosa cells and follicular fluid. All such components play critical roles in the development of competent oocytes and in the formation of high quality embryos (Dumesic et al., 2015; Sirard et al., 2006). The dynamics in this environment is highly orchestrated through the messengers in the follicular fluid such as miRNAs, cytokines, hormones, peptides and other molecules (Revelli et al., 2009). These molecules are considered as potential biomarkers for oocyte quality and assisted reproduction treatment (ART) outcomes (Dumesic et al., 2015; Wallace et al., 2012).

Glycodelin (Gd) is a glycoprotein from the lipocalin superfamily. It is synthesized in the endometrium in response to hormones progesterone and relaxin (Halttunen et al., 2000). Gd has three identified isoforms that share a similar protein core, but contain different glycan side chains. These Gd isoforms are expressed in different locations in the reproductive system such as the amniotic fluid ( $G d A$ ), the endometrium ( $\mathrm{Gd} \mathrm{A}$ ), seminal plasma ( $\mathrm{Gd} \mathrm{S}$ ), and follicular fluid (Gd F) (Seppälä et al., 2002). Additionally, cumulus cells are able to convert exogenous $\mathrm{Gd} \mathrm{A}$ and $\mathrm{F}$ to $\mathrm{Gd} \mathrm{C}$, thus enhancing the zona binding capacity of spermatozoa (Chiu et al., 2007). Although Gd is primarily synthesized in the reproductive tract, its expression was also detected in other tissues such as the bone marrow (Kämäräinen et al., 1994) and gynecological tumors (Horowitz et al., 2001; Kämäräinen et al., 1996).

The importance of follicular fluid Gd levels in the timing of fertilization was previously described in a report demonstrating that Gd A and Gd F dose dependently inhibited fertilization by blocking the binding of sperm to the Zona Pellucida (Chiu et al., 2003; Oehninger et al., 1995). In contrast, $\mathrm{Gd} C$ favors fertilization by enhancing spermatozoa-Zona Pellucida binding (Chiu et al., 2007). Furthermore, it is known that GD mRNA is expressed in the secondary late follicles and protein Gd is taken up by the cumulus cells (Tse et al., 2002). The association between $\mathrm{Gd}$ and fertilization is well defined. However, the possible effects of follicular Gd on the competence of the developing oocyte have not been studied yet. Moreover, it is unclear whether alterations in the follicular fluid Gd levels affect subsequent embryo development.

This study aimed to investigate whether follicular fluid Gd levels have an effect on the quality of early cleavage stage embryos. Thus, we measured Gd levels in the follicular fluid of patients undergoing ART and assessed the correlation between Gd levels, embryo development and treatment outcome.

\section{MATERIALS AND METHOD}

\section{Study design and patient selection}

This study enrolled 176 couples undergoing assisted reproduction treatment between April 2009 and October 2012 in the Department of Obstetrics and Gynecology of the School of Medicine of Istanbul University. The inclusion criteria were: (1) presence of only one cause of infertility without additional indicators of female or male infertility; (2) absence of history of endometriosis confirmed with laparoscopic surgery; (3) absence of advanced maternal age and excess follicle stimulating hormone (FSH) levels.

Thirty-one couples were excluded from the study for not meeting the inclusion criteria. Three were diagnosed with endometriosis, two had advanced maternal age ( $>40$ years old), five had exceedingly high FSH levels ( $>20 \mathrm{mIU} / \mathrm{ml}$ ), and 21 had undergone testicular biopsy for severe male infertility. As a result, 145 couples were included in the study. Cases of female infertility were diagnosed as ovulatory factor $(n=14)$, tubal factor $(n=18)$, and unexplained infertility $(n=57)$. Fifty-six couples were diagnosed with male factor infertility only. All participants volunteered for the study and signed informed consent terms. The Ethics Committee of the School of Medicine of Istanbul University approved the study ( $\left.\mathrm{N}^{\circ}: 1228\right)$. 
Controlled ovarian hyperstimulation and assisted reproduction techniques

Controlled ovarian hyperstimulation $(\mathrm{COH})$ was performed with standard gonadotropin hormone in antagonist or long agonist protocols. In antagonist protocols, recombinant FSH (Gonal F, Serono) was injected daily starting from day 3 of the menstrual cycle in combination with a fixed dose of a GnRH antagonist (Cetrorelix, Cetrotide, Serono, $0.25 \mathrm{mg}$ ) starting from day 6 of stimulation until human chorionic gonadotropin (hCG) administration. In long agonist protocols, a gonadotropin releasing hormone $(\mathrm{GnRH})$ agonist (leuprolide acetate, Lucrin, Abbot Laboratories or triptorelin acetate, Decapeptyl, Ferring, 0.1 $\mathrm{mg}$ ) was administered daily starting on day 21 (mid-luteal phase) of the previous cycle. After pituitary down regulation, the dose of agonist was decreased to $0.05 \mathrm{mg}$ and patients received recombinant FSH (Gonal F, Serono) until human chorionic gonadotropin (hCG) administration. Ultrasound examination and serum estradiol levels were used in the assessment of follicular development. Transvaginal follicular aspiration was performed 36 hours after human chorionic gonadotropin (Pregnyl, Organon, 10,000 IU) administration.

Fertilization was performed by intracytoplasmic sperm injection (ICSI) and fertilization control was done 16-18 hours after ICSI. The quality of the resulting embryos was graded daily according to the laboratory standards modified from the ALPHA and ESHRE (European Society for Reproductive Medicine and Embryology) Guidelines (Alpha Scientists in Reproductive Medicine \& ESHRE Special Interest Group of Embryology, 2011), in which Grade I (GI) included top quality embryos and Grade III (GIII) featured lower quality embryos. Embryo culture was performed in sequential mediums (G1 and G2 medium, Vitrolife) and embryos were incubated in a humidified incubator at $37^{\circ} \mathrm{C}$ with $5 \% \mathrm{CO}_{2}$ in the air.

Elective single-embryo or double-embryo transfers were conducted on day three of fertilization under ultrasound visualization with a soft transfer catheter. The number of embryos transferred was determined based on the regulations set out by the Turkish Ministry of Health. Implantation was confirmed when a positive $B$-human chorionic gonadotropin ( $\beta-\mathrm{hCG}$ ) level (>10 IU/mL) was detected 12 days after embryo transfer. Pregnancy was confirmed by the verification of a fetal heartbeat on ultrasound examination.

\section{Sample collection}

As a part of assisted reproduction treatment, follicular fluids (FF) were obtained from mature follicles measuring $18-25 \mathrm{~mm}$ in diameter at the day of oocyte retrieval, and were immediately transferred to sterile $15 \mathrm{ml}$ conical tubes. Subsequently, FF samples were centrifuged at $400 \mathrm{~g}$ for 15 minutes and the supernatants were stored in liquid nitrogen for further analysis. Blood samples were drawn on day three of menstruation for hormonal profile tests including luteinizing hormone ( $\mathrm{LH}), \mathrm{FSH}$, estradiol, and progesterone.

\section{Measurement of glycodelin levels by ELISA}

Gd concentrations of the FF samples obtained from mature follicles were assessed with enzyme-linked immunosorbent assay test kits (human glycodelin ELISA kit, CUSABIO, China) with a sensitivity of $0.195 \mathrm{ng} / \mathrm{ml}$, a detection range of $0.78-50 \mathrm{ng} / \mathrm{ml}$, an intra-assay coefficient variation $(\mathrm{CV})<8 \%$ and an inter-assay CV $<10 \%$. Samples were analyzed in duplicates and mean readings were expressed in $\mathrm{ng} / \mathrm{ml}$.

\section{Statistical methods}

Analysis of variance (ANOVA) was used to compare between continuous variables such as age, body mass index (BMI), hormone levels on day 3 of menstruation, number of oocytes, fertilization, number of grade I and grade III embryos on day 3, number of arrested embryos, and glycodelin levels. Categorical variables such as implantation, pregnancy, and type of induction protocols were compared using the chi-square test. Correlation analysis was based on Spearman's rank correlation coefficient. $p$-values $<0.05$ were considered statistically significant. Values were given as means and standard deviations (SD). All statistical analyses were performed using Windows SPSS version 14.0 software (SPSS Inc., Chicago, IL, USA).

\section{RESULTS}

\section{Patient characteristics and follicular fluid gly- codelin levels}

In the overall study group, mean female and male ages were $31.58( \pm 4.62 ; 20-40)$ and $34.73( \pm 5.31 ; 25-52)$ respectively. In order to investigate the possible association of Gd among different female infertility groups, we compared the follicular fluid Gd levels of groups diagnosed with ovulatory factor, tubal factor, and unexplained infertility. However, there were no statistically significant differences in Gd levels between different female infertility groups (ovulatory factor vs. unexplained infertility (6.91 vs. 6.53, $p=0.79)$, tubal factor vs. unexplained infertility (7.82 vs. $6.53, p=0.26$ ) and ovulatory factor vs. tubal factor (7.82 vs. $6.91, p=0.59)$ ). Additionally, Gd levels were not different between female (ovulatory and tubal factor) and male factor groups $(p=0.76)$. Table 1 presents the patient characteristics and ART outcomes categorized by cause of infertility.

Although $\mathrm{Gd}$ secretion is induced by progesterone in the endometrium, no correlation was observed between these molecules in our data $(p=0.14)$. However, higher Gd levels were correlated with higher D3 FSH levels $(r=0.18, p=0.04)$. FSH levels were also correlated with age $(r=0.18, p=0.04)$, although there was no correlation between female age and $\mathrm{Gd}$ levels $(p=0.36)$. Additionally, there seems to be an inverse correlation between Gd levels and the number of mature follicles monitored on oocyte retrieval day $(r=-0.16, p=0.06)$.

In order to test whether the ovulation induction protocol had an effect on Gd levels and ART outcomes, the outcomes of patients induced with antagonist $(n=126)$ and long agonist $(n=18)$ protocols were compared, although the latter group had a smaller sample size. However, no differences were seen between the antagonist and long protocol ovulation induction groups in terms of fertilization rates $(p=0.97)$, implantation rates $(p=0.66)$, developmental arrest up to day $3(p=0.17)$, grade I embryo development on day $3(p=0.49)$, and Gd concentrations $(p=0.77)$ (Table 2$)$.

In vitro embryo development and glycodelin levels

Gd levels were negatively correlated with the number of high quality embryos on day $3(r=-0.20, p=0.05)$. The number of high quality embryos was negatively correlated with increasing FSH levels $(r=-0.27, p=0.008)$. On the other hand, in one-way ANOVA analysis, Gd levels were not associated with implantation (implantation $6.74( \pm 4.36)$ vs. no implantation $7.07( \pm 4.50), p=0.67)$ or ongoing pregnancy rates (pregnancy $7.15( \pm 4.51)$ vs. non-pregnancy $7.07( \pm 4.49), p=0.99)$ in the study group. Additionally, there was no statistically significant difference in $\mathrm{Gd}$ levels between biochemical and clinical pregnancies (7.15 ( \pm 4.52$)$ vs. $5.11( \pm 3.38), p=0.19)$. 
Table 1. Patient characteristics and ART outcomes categorized according to cause of infertility

\begin{tabular}{|c|c|c|c|c|c|}
\hline \multirow{2}{*}{ Characteristics } & Ovulatory Factor & Tubal Factor & Unexplained Infertility & Male Factor & \\
\hline & $n=14$ & $n=18$ & $n=57$ & $n=56$ & $p$ value \\
\hline Female age (years) & $33.36( \pm 4.73)$ & $32.17( \pm 4.18)$ & $32.19( \pm 4.72)$ & $30.32( \pm 4.42)$ & 0.05 \\
\hline Male age (years) & $36.36( \pm 6.37)$ & $36.94( \pm 5.80)$ & $34.88( \pm 5.17)$ & $33.46( \pm 4.77)$ & 0.05 \\
\hline BMI $\left(\mathrm{kg} / \mathrm{m}^{2}\right)$ & $24.87( \pm 5.81)$ & $26.18( \pm 5.33)$ & $25.79( \pm 4.73)$ & $24.84( \pm 3.15)$ & 0.63 \\
\hline $\mathrm{FSH}(\mathrm{mIU} / \mathrm{mL})$ & $8.01( \pm 6.17)$ & $7.40( \pm 2.82)$ & $7.51( \pm 3.02)$ & $6.97( \pm 2.10)$ & 0.67 \\
\hline $\mathrm{LH}(\mathrm{IU} / \mathrm{mL})$ & $4.74( \pm 4.22)$ & $7.12( \pm 8.16)$ & $5.71( \pm 2.92)$ & $5.41( \pm 1.92)$ & 0.33 \\
\hline Estradiol $(\mathrm{pg} / \mathrm{mL})$ & $40.61( \pm 22.38)$ & $57.66( \pm 30.54)$ & $45.18( \pm 16.48)$ & $47.22( \pm 22.23)$ & 0.13 \\
\hline Progesterone (ng/mL) & $0.39( \pm 0.19)$ & $0.40( \pm 0.17)$ & $0.48( \pm 0.21)$ & $0.71( \pm 0.88)$ & 0.39 \\
\hline Total AFC (n) & $5.00( \pm 2.21)$ & $6.93( \pm 3.37)$ & $9.15( \pm 7.09)$ & $8.60( \pm 4.91)$ & 0.07 \\
\hline Glycodelin (ng/mL) & $6.91( \pm 6.53)$ & $7.82( \pm 4.02)$ & $6.53( \pm 4.32)$ & $7.12( \pm 4.06)$ & 0.73 \\
\hline MII (n) & $3.57( \pm 3.59)$ & $6.44( \pm 4.62)$ & $7.42( \pm 5.04)$ & $8.23( \pm 4.80)$ & 0.01 \\
\hline Fertilization (yes,\%) & $85.71( \pm 24.15)$ & $74.00( \pm 19.55)$ & $81.67( \pm 16.45)$ & $73.10( \pm 24.27)$ & 0.06 \\
\hline GI Embryo (n) & $1.33( \pm 1.87)$ & $1.47( \pm 1.60)$ & $2.45( \pm 2.91)$ & $2.42( \pm 2.18)$ & 0.36 \\
\hline Arrest $(n)$ & $0.57( \pm 0.76)$ & $0.76( \pm 1.72)$ & $1.04( \pm 1.69)$ & $1.08( \pm 1.64)$ & 0.69 \\
\hline Implantation (yes,\%) & 21.4 & 22.2 & 36.8 & 40.0 & 0.37 \\
\hline Pregnancy* $($ yes, $\%)$ & 14.3 & 11.1 & 31.6 & 32.7 & 0.50 \\
\hline
\end{tabular}

Values are expressed as mean \pm SD. BMI, body mass index. AFC, antral follicle count.

MII, metaphase II oocytes. *Pregnancy indicates clinical pregnancy rates.

Table 2. Patient characteristics and ART outcomes in different ovarian hyperstimulation protocol groups

\begin{tabular}{|l|c|c|c|}
\hline \multirow{2}{*}{ Thable 2. Patient characteristics and ART outcomes in different ovarian hyperstimulation protocol groups } \\
\cline { 2 - 4 } & Antagonist Protocol & Long Agonist Protocol & $\mathbf{n = 1 8}$ \\
\hline Female age (years) & $\mathbf{n = 1 2 6}$ & $30.11( \pm 3.50)$ & value \\
\hline Male age (years) & $31.79( \pm 4.75)$ & $33.33( \pm 5.33)$ & 0.15 \\
\hline BMI $\left(\mathrm{kg} / \mathrm{m}^{2}\right)$ & $34.94( \pm 5.32)$ & $24.61( \pm 4.08)$ & 0.23 \\
\hline FSH $(\mathrm{mIU} / \mathrm{mL})$ & $25.45( \pm 4.3)$ & $8.20( \pm 4.45)$ & 0.49 \\
\hline LH $(\mathrm{IU} / \mathrm{mL})$ & $7.25( \pm 2.94)$ & $5.65( \pm 2.06)$ & 0.96 \\
\hline Glycodelin $(\mathrm{ng} / \mathrm{mL})$ & $5.70( \pm 3.96)$ & $6.68( \pm 4.02)$ & 0.77 \\
\hline MII $(\mathrm{n})$ & $7.01( \pm 4.50)$ & $10.61( \pm 5.65)$ & 0.002 \\
\hline Fertilization $(\%)$ & $6.79( \pm 4.63)$ & $78.11( \pm 17.58)$ & 0.97 \\
\hline GI Embryo $(\mathrm{n})$ & $77.88( \pm 21.78)$ & $2.70( \pm 2.58)$ & 0.49 \\
\hline Arrest $(\mathrm{n})$ & $2.13( \pm 2.14)$ & $1.50( \pm 1.97)$ & 0.17 \\
\hline Implantation $(y e s, \%)$ & $0.91( \pm 1.54)$ & 38.9 & 0.66 \\
\hline Pregnancy* (yes, \%) & 33.6 & 27.8 & 0.75 \\
\hline
\end{tabular}

Values are expressed as mean \pm SD.

BMI, body mass index. MII, metaphase II oocytes. Pregnancy indicates clinical pregnancy rates.

\section{DISCUSSION}

Follicular fluid is one of the main components of the microenvironment of developing oocytes. Certain molecules in follicular fluid have been previously identified as biomarkers for oocyte quality and subsequent embryo development (Dumesic et al., 2015, Wallace et al., 2012). In this study, we evaluated glycodelin levels in the follicular fluid of patients undergoing assisted reproductive treatment and observed that higher Gd levels were negatively correlated with the number of high quality embryos in vitro.
ICSI is one of the fertilization techniques used in ART. In this procedure, the oocyte is retrieved by follicular puncture and subsequently removed from its follicular components (follicular fluid and surrounding granulosa cells). Thus, between oocyte retrieval and fertilization by the microinjection of sperm, oocytes resting in cell culture medium are isolated from their natural environment and from the effects of follicular fluid molecules. Therefore, the impact of Gd on fertilization and embryo development in ICSI-derived embryos might reflect the influence of follicular 
Gd on the developing oocyte. Our finding regarding the negative correlation between Gd levels and number of high quality embryos obtained in ART cycles suggests that follicular levels of $\mathrm{Gd}$ might influence the development of competent oocytes. Moreover, basal FSH levels were positively correlated with FF Gd levels and negatively correlated with the number high quality embryos. These findings might suggest that with the diminishing ovarian reserve and alterations in the follicular content including Gd levels, the number of oocytes that might potentially develop into healthy embryos also diminishes. Similarly, Zak et al. (2010) demonstrated that Gd levels in FF from unfertilized oocytes were higher than in fertilized oocytes. The authors proposed that these results might indicate the effect of Gd on fertilization and implantation (Zak et al., 2010). However, they suggested that the choice of fertilization technique influenced the fertilization rate and the number of total embryos developed (fertilized oocytes on day 1), with higher Gd levels negatively correlated with IVF outcomes but not with ICSI outcomes. In our study, no correlation was seen with fertilization rates; however, Gd levels were negatively correlated with ICSI-derived good quality embryos on day 3 . This conflict between studies might have arisen from the different time points (day 1 vs. day 3 ) at which developing embryos were assessed.

Follicular fluid is the main source of Gd F other than the fallopian tube (Chiu et al., 2003). In the ovary, Gd protein was first demonstrated by Immunohistochemical analysis in the stromal cells of the ovarian cortex, theca interna, and granulosa cells in the follicular phase. Additionally, Gd was detected in the theca interna of the corpus luteum and luteinized granulosa cells in the luteal phase (Kämäräinen et al., 1996). However, Gd levels in follicular fluid and granulosa cells were found to be lower than in amniotic fluid and decidualized endometrium (Tse et al., 2002). Furthermore, glycodelin mRNA expression was restricted to luteinized granulosa cells, with no expression detected in cumulus cells (Tse et al., 2002). No significant differences were found between the groups included in a recent study comparing follicular fluid and serum Gd levels between ART patients induced with long-GnRH agonist and antagonist protocols (Aydin et al., 2014). Additionally, the same authors reported in another study that FF Gd concentrations were not statistically different between clinically pregnant and non-pregnant patients, and that no significant differences were found in Gd levels between the early follicular and preovulatory phases. However, on the day of oocyte retrieval, serum Gd levels were significantly higher than in the early follicular phase (Hassa et al., 2015). In line with these studies, we saw no difference in Gd levels between the $\mathrm{GnRH}$ agonist and antagonist ovulation induction groups. Additionally, we found that Gd was not predictive for implantation or pregnancy rates in assisted reproduction. On the other hand, a study with a small sample size (Zak et al., 2010) demonstrated that the FF Gd levels of patients undergoing ART was significantly higher among patients with implantation failure.

Although timing of Gd secretion was investigated in serum (Bersinger et al., 2009), amniotic fluid (Riittinen et al., 1989), the endometrium (Amir et al., 2009; Bersinger et al., 2008), the fallopian tubes (Amir et al., 2009), and in follicular cells (Tse et al., 2002), there is no clear data comparing the expression levels or timing of expression of Gd subtypes throughout the female genital tract. Gd A has been the most investigated Gd type. Although undetectable around ovulation period, the highest expression of the Gd transcript was detected within the mid-luteal phase (around D2-D7) in endometrium samples on different microarray and quantitative real time PCR (qPCR) studies (Bersinger et al., 2008; Horcajadas et al., 2005; Mirkin et al., 2005; Riesewijk et al., 2003; Kao et al., 2002). This period overlaps with the "implantation window", a very short period of time in which embryos are permitted to successfully attach to uterine tissue. In a study conducted to detect serum levels of $\mathrm{Gd}$, lowest levels were observed around ovulation whereas highest levels were detected at the end of the luteal phase, with serum glycodelin peaking around 8 days after the progesterone peak (Bersinger et al., 2009). Based on earlier studies reporting a correlation between higher progesterone levels and higher Gd levels during the menstrual phase (Seppälä et al., 1994; Joshi, 1983 ) and subsequent in vivo and in vitro studies (Taylor et al., 2000; Vaisse et al., 1990; Julkunen et al., 1986), endometrial $\mathrm{Gd}$ secretion was associated with progesterone. Several putative glucocorticoid/progesterone-responsive elements (GRE/PREs) have been identified in the gene coding protein Gd (progestagen-associated endometrial protein, PAEP or GD) (Vaisse et al., 1990); however, it is not clear whether progesterone is directly involved in the expression and release of Gd in the endometrium or whether Gd secretion is primarily modulated by other molecules secreted in the luteal phase such as relaxin (Seppälä et al., 2002; Tse et al., 2002; Stewart et al., 1997). Our study found no correlation between basal serum progesterone and FF Gd levels in ART patients. However, Gd levels were detected from luteinized follicles when sampling was performed during the oocyte retrieval procedure following 2336 hours of hCG injection and progesterone measurements were performed on the follicular phase (day 3 of menstrual cycle). This might be a reason why we were unable to observe a correlation between progesterone and Gd levels.

A limitation of our study was FF Gd levels were not compared to serum Gd levels. Another limitation was that Gd and hormone levels were obtained at different times in follicular development.

\section{CONCLUSION}

This study investigated the follicular effect of Gd levels on preimplantation embryo development and on the outcomes of assisted reproduction treatment. To our knowledge, this is the first report demonstrating a correlation between follicular Gd levels and quality of in vitro developed embryos (D3 embryos). However, the exact cellular mechanisms by which Gds impacts the development of competent oocytes and subsequent embryo development remain to be elucidated.

\section{ACKNOWLEDGEMENT}

This study received support from the Scientific Research Projects Coordination Unit of Istanbul University (Project number: 25065).

\section{CONFLICT OF INTEREST}

The authors have no conflict of interest to declare.

\section{Corresponding Author:}

Bilge Ozsait Selcuk

Istanbul University, Istanbul, Turkey

Institute for Experimental Medicine

Department of Genetics

Istanbul, Turkey

E-mail: ozsaitb@istanbul.edu.tr

\section{REFERENCES}

Alpha Scientists in Reproductive Medicine and ESHRE Special Interest Group of Embryology. The Istanbul consensus workshop on embryo assessment: proceedings of an expert meeting. Hum Reprod. 2011;26:1270-83. PMID: 21502182 DOI: 10.1093/humrep/der037 
Amir M, Romano S, Goldman S, Shalev E. Plexin-B1, glycodelin and MMP7 expression in the human fallopian tube and in the endometrium. Reprod Biol Endocrinol. 2009;7:152. PMID: 2004008 DOI: 10.1186/1477-7827$7-152$

Aydin $\mathrm{Y}$, Hassa $\mathrm{H}$, Isikci T, Colak O. Follicular fluid and serum vascular endothelial growth factor, interleukin (IL)$1 \beta$ and glycodelin concentrations: comparison between long-gonadotropin-releasing hormone ( $\mathrm{GnRH})$-agonist and GnRH-antagonist cycles: a randomized controlled trial. Gynecol Endocrinol. 2014;30:734-8. PMID: 24927078 DOI: $10.3109 / 09513590.2014 .929655$

Bersinger NA, Wunder DM, Birkhäuser MH, Mueller MD. Gene expression in cultured endometrium from women with different outcomes following IVF. Mol Hum Reprod. 2008;14:475-84. PMID: 18539642 DOI: $10.1093 / \mathrm{molehr} /$ gan036

Bersinger NA, Birkhäuser MH, Yared M, Wunder DM. Serum glycodelin pattern during the menstrual cycle in healthy young women. Acta Obstet Gynecol Scand. 2009;88:121521. PMID: 19900139 DOI: 10.3109/00016340903294264

Chiu PC, Koistinen R, Koistinen H, Seppala M, Lee KF, Yeung WS. Zona-binding inhibitory factor-1 from human follicular fluid is an isoform of glycodelin. Biol Reprod. 2003;69:36572. PMID: 12672671 DOI: $10.1095 /$ biolreprod.102.012658

Chiu PC, Chung MK, Koistinen R, Koistinen $H$, Seppala $\mathrm{M}$, Ho PC, Ng EH, Lee KF, Yeung WS. Cumulus oophorus-associated glycodelin-C displaces sperm-bound glycodelin-A and $-\mathrm{F}$ and stimulates spermatozoa-zona pellucida binding. J Biol Chem. 2007;282:5378-88. DOI: 10.1074/jbc. M607482200

Dumesic DA, Meldrum DR, Katz-Jaffe MG, Krisher RL, Schoolcraft WB. Oocyte environment: follicular fluid and cumulus cells are critical for oocyte health. Fertil Steril. 2015;103:303-16. PMID: 25497448 DOI: $10.1016 /$ j.fertnstert.2014.11.015

Halttunen $M$, Kämäräinen $M$, Koistinen $H$. Glycodelin: a reproduction-related lipocalin. Biochim Biophys Acta. 2000;1482:149-56. PMID: 11058757 DOI: 10.1016/ S0167-4838(00)00158-8

Hassa H, Aydin Y, Isikci T, Oner S. Follicular phase serum and follicular fluid glycodelin measurements in gonadotropin-releasing hormone (GnRH)-antagonist assisted reproduction cycles: A prospective cohort study. Clin Exp Obstet Gynecol. 2015;42:367-71. PMID: 26152013

Horcajadas JA, Riesewijk A, Polman J, van Os R, Pellicer A, Mosselman S, Simon C. Effect of controlled ovarian hyperstimulation in IVF on endometrial gene expression profiles. Mol Hum Reprod. 2005;11:195-205. PMID: 15695772 DOI: $10.1093 /$ molehr/gah150

Horowitz IR, Cho C, Song M, Flowers LC, Santanam $\mathrm{N}$, Parthasarathy $S$, Ramachandran $S$. Increased glycodelin levels in gynecological malignancies. Int J Gynecol Cancer. 2001;11:173-9. PMID: 11437921 DOI: 10.1046/j.1525-1438.2001.01017.x
Joshi SG. A progestagen-associated protein of the human endometrium: basic studies and potential clinical applications. J Steroid Biochem. 1983;19:751-7. PMID: 6411990 DOI: $10.1016 / 0022-4731(83) 90007-9$

Julkunen M, Apter D, Seppälä M, Stenman UH, Bohn H. Serum levels of placental protein 14 reflect ovulation in nonconceptional menstrual cycles. Fertil Steril. 1986;45:47-50. PMID: 3943649 DOI: 10.1016/S0015-0282(16)49095-3

Kämäräinen M, Riittinen L, Seppälä M, Palotie A, Andersson LC. Progesterone-associated endometrial protein--a constitutive marker of human erythroid precursors. Blood. 1994;84:467-73. PMID: 8025274

Kämäräinen $M$, Leivo I, Koistinen $R$, Julkunen $M$, Karvonen $U$, Rutanen EM, Seppälä M. Normal human ovary and ovarian tumors express glycodelin, a glycoprotein with immunosuppressive and contraceptive properties. Am J Pathol. 1996;148:1435-43. PMID: 8623915

Kao LC, Tulac S, Lobo S, Imani B, Yang JP, Germeyer A, Osteen K, Taylor RN, Lessey BA, Giudice LC. Global gene profiling in human endometrium during the window of implantation. Endocrinology. 2002;143:2119-38. PMID: 12021176 DOI: $10.1210 /$ endo.143.6.8885

Mirkin S, Arslan M, Churikov D, Corica A, Diaz JI, Williams $S$, Bocca $S$, Oehninger $S$. In search of candidate genes critically expressed in the human endometrium during the window of implantation. Hum Reprod. 2005;20:2104-17. PMID: 15878921 DOI: 10.1093/humrep/dei051

Oehninger S, Coddington CC, Hodgen GD, Seppälä M. Factors affecting fertilization: endometrial placental protein 14 reduces the capacity of human spermatozoa to bind to the human zona pellucida. Fertil Steril. 1995;63:377-83. PMID: 7531163 DOI: 10.1016/S0015-0282(16)57372-5

Revelli A, Delle Piane L, Casano S, Molinari E, Massobrio M, Rinaudo P. Follicular fluid content and oocyte quality: from single biochemical markers to metabolomics. Reprod Biol Endocrinol. 2009;7:40. PMID: 19413899 DOI: 10.1186/1477-7827-7-40

Riesewijk A, Martín J, van Os R, Horcajadas JA, Polman J, Pellicer A. Mosselman S, Simón C. Gene expression profiling of human endometrial receptivity on days $\mathrm{LH}+2$ versus $\mathrm{LH}+7$ by microarray technology. Mol Hum Reprod. 2003;9:25364. PMID: 12728018 DOI: $10.1093 / \mathrm{molehr} / g a g 037$

Riittinen L, Julkunen M, Seppälä M, Koistinen R, Huhtala ML. Purification and characterization of endometrial PP14 from mid-trimester amniotic fluid. Clin Chim Acta. 1989;184:1929. PMID: 2688994 DOI: 10.1016/0009-8981(89)90253-2

Seppälä M, Koistinen R, Rutanen EM. Uterine endocrinology and paracrinology: insulin-like growth factor binding protein-1 and placental protein 14 revisited. Hum Reprod. 1994;9:917-25. PMID: 7523437 DOI: 10.1093/oxfordjournals. humrep.a138617

Seppälä M, Taylor RN, Koistinen H, Koistinen R, Milgrom E. Glycodelin: a major lipocalin protein of the reproductive axis with diverse actions in cell recognition and differentiation. Endocr Rev. 2002;23:401-30. PMID: 12202458 DOI: 10.1210/er.2001-0026 
Sirard MA, Richard F, Blondin P, Robert C. Contribution of the oocyte to embryo quality. Theriogenology. 2006;65:126-36. PMID: 16256189 DOI: $10.1016 / j$.theriogenology.2005.09.020

Stewart DR, Erikson MS, Erikson ME, Nakajima ST, Overstreet JW, Lasley BL, Amento EP, Seppala M. The role of relaxin in glycodelin secretion. J Clin Endocrinol Metab. 1997;82:839-46. PMID: 9062493 DOI: $10.1210 /$ jc. 82.3 .839

Taylor RN, Vigne JL, Zhang P, Hoang P, Lebovic DI, Mueller MD. Effects of progestins and relaxin on glycodelin gene expression in human endometrial cells. Am J Obstet Gynecol. 2000;182:841-7. PMID: 10764460

Tse JY, Chiu PC, Lee KF, Seppala M, Koistinen H, Koistinen $R$, Yao YQ, Yeung WS. The synthesis and fate of glycodelin in human ovary during folliculogenesis. Mol Hum Reprod. 2002;8:142-8. PMID: 11818517 DOI: $10.1093 /$ molehr/8.2.142
Vaisse C, Atger M, Potier B, Milgrom E. Human placental protein 14 gene: sequence and characterization of a short duplication. DNA Cell Biol. 1990;9:401-13. PMID: 2206398 DOI: $10.1089 /$ dna.1990.9.401

Wallace M, Cottell E, Gibney MJ, McAuliffe FM, Wingfield M, Brennan L. An investigation into the relationship between the metabolic profile of follicular fluid, oocyte developmental potential, and implantation outcome. Fertil Steril. 2012;97:1078-84. PMID: 22365382 DOI: 10.1016/j.fertnstert.2012.01.122

Zak T, Kaczmarek-Sroka A, Kubiaczyk B, Wirstlein P, Breborowicz GH. Impact of follicular fluid glycodelin on the process of fertilization and implantation after the transfer of embryos in the process of in vitro fertilization (IVF). Arch Perinat Med. 2010;16:234-7 